\title{
REMOTE SENSING DATA FUSION TO DETECT ILLICIT CROPS AND UNAUTHORIZED AIRSTRIPS
}

\author{
J. A. Pena ${ }^{1}$, T. Yumin ${ }^{1}$, Huaqing Liu ${ }^{1}$, Binbin Zhao ${ }^{2}$ J. A. Garcia ${ }^{3}$, J. Pinto ${ }^{4}$ \\ ${ }^{1}$ School of Transportation Science \& Engineering, Beihang University Beijing, China - jpena@abae.gob.ve, \\ tanym@buaa.edu.cn, yhye@buaa.edu.cn \\ ${ }^{2}$ China Electric Power Research Institute, Beijing, China - 123zhaobinbin@163.com \\ ${ }^{3}$ Russian State Hidrometeorological University, St. Petesburgo, Russia - j_escalona26@ hotmail.com \\ ${ }^{4}$ National Anti-drug Office of Venezuela - jpintos@abae.gob.ve
}

\section{Commission WG III/6 Remote Sensing Data Fusion}

KEY WORDS: Remote sensing, Data fusion, Illicit crops, Unauthorized Airstrip.

\begin{abstract}
:
Remote sensing data fusion has been playing a more and more important role in crop planting area monitoring, especially for crop area information acquisition. Multi-temporal data and multi-spectral time series are two major aspects for improving crop identification accuracy. Remote sensing fusion provides high quality multi-spectral and panchromatic images in terms of spectral and spatial information, respectively. In this paper, we take one step further and prove the application of remote sensing data fusion in detecting illicit crop through LSMM, GOBIA, and MCE analyzing of strategic information. This methodology emerges as a complementary and effective strategy to control and eradicate illicit crops.
\end{abstract}

\section{INTRODUCTION}

Data fusion, sensor fusion, information fusion, sensor data fusion, or simply fusion - no matter which specific term is used, refers to the science of combining measurements, signals, or observations from different sources to obtain a result that is in some sense better than what could have been achieve without this combination is a widely discussed topic in many disciplines (Schmitt et al., 2016). In the context of remote sensing, data fusion is usually used, although sometimes this term extended to sensor data fusion if data from different sensor types are used, and sometimes this is termed image fusion if only twodimensional images are combined. (Gustafsson et al., 2012). Multi-sensor data fusion is a technology to enable combining information from several sources to form a unified picture, leaves a great deal of room for interpretation and adaptation in certain application fields. (Khaleghi et al., 2013).

Remote Sensing (RS) has been playing a more and more important role in crop planting area monitoring, especially for crop area information acquisition (Liu et al., 2015; Chen et al., 2005) Multi-temporal data (Wang et al., 2015; Zhang et al., 2012) and multi-spectral (Zhao, 2014; Lin et al., 2010) time series are two major aspects for improving crop identification accuracy. Different sensors have their own characteristics in terms of their revisit periods and spectral bands. As will be demonstrated in this study, an optimal analysis and selection of these specific parameters will promote operational acquisition of crop area information.

However, it is necessary to consider that the identification and detection of activities associated with illicit crops from satellite images is not an easy task, since they are constituted by a mixture of elements common in nature, predominating the soil, vegetation and water, in addition to being affected by different environmental conditions.

\subsection{Background}

To the West of The Bolivarian Republic of Venezuela, it borders with the Republic of Colombia forming a border extension of $2,219 \mathrm{~km}$, in the south with the Federative
Republic of Brazil, with a border extension of $1,700 \mathrm{~km}$, without including the Essequibo territory; To the east with the Atlantic Ocean having an area of 65,578 km2 and Guyana with a border extension of $743 \mathrm{~km}$ (currently zone in claim comprising $159,500 \mathrm{~km} 2$ ), for a total perimeter in limits of $7,870 \mathrm{~km}$ and an extension of continental territory of 916,445 km2. (IGVSB, 2017).

Coca cultivation occurs along Venezuela's border with Colombia, which is taken as illicit crop worldwide. The activities associated with illicit crops, without a doubt, negatively affect the comprehensive security of the nation, where this causes problems mainly in the economic, social, political, cultural, geographical and environmental fields. However, one of the key aspects of the detection of elements linked to illicit crops is the availability of up-to-date information on the geographical area.

\subsection{Prior works}

It is notable that a significant portion of the literature about the fusion of remote sensing imagery is based on either image fusion for pan-sharpening purposes or feature fusion for the improvement of classification results for example, Ehlers et al.,(2010)provided a comprehensive overview of pansharpening methods based on well-known algorithms such as the Brovey transform; Comprehensive and recent reviews of the research activities in remote sensing data fusion with special regard for the mapping of urban areas are provided in (Gamba,2013). Another important development is the introduction of spectral-spatial fusion techniques, which paved the way for even better pan-sharpening techniques and improved classification results from multispectral data (Huang et al., 2012; Zhang et al., 2012; Huang et al., 2013; Melgani, 2002) Only a handful of contributions in the literature have addressed the use of the fusion of remote sensing with government and military data to detect illicit crop.

Similarly, there are other researches related to the identification and eradication of illicit crops assessing the effectiveness of this activities yields contradictory conclusions. Results vary depending on the period of time evaluated and the unit of 
analysis (Davalos, 2016). The methods used to identify and eradicate crops- have little impact on illicit crops or increases the area under coca cultivation (Bogliacino et al., 2012; Moreno et al., 2003; Reyes, 2011).

In this research we investigate the potential model for remote sensing data fusion with strategic information. The proposed model uses LSMM and Prodes methodology to extract the spectral-spatial features with the fraction images; GOBIA with multiresolution segmentation algorithm to identify the Potential areas for planting of illicit crops and MCE to establish existing relationships between geographic space and activities related to illicit crops.

\section{METHODOLOGY}

\subsection{Data fusion}

Image fusion is the combination of two or more different images to form a new image by using a certain algorithm. On the basis of image-centered reviews like this one or the work by (Zhang, 2010) we see that a certain emphasis in remote sensing data fusion is placed on image interpretation, either in the context of classification tasks or in the context of pan sharpening. In the context of classification and pan sharpening, we choose the combination of VRSS-2 (Venezuelan Remote Sensing Satellites 2) and LANDSAT-8 in this research. An important aspect of the research was the use of strategic information of the Venezuelan National Armed Forces relating to the previous military operations that have been carried out for the eradication of these illicit crops. This data (pictures, photos, videos, procedures, etc) help as auxiliary data for the development of this methodology in this paper.

\section{$2.2 \quad$ LSMM}

Mixed pixels exist commonly in coarse spatial resolution images (Keshava et al., 2002). Spectral unmixing is the process of decomposing the spectral signature of a mixed pixel into a set of endmembers and their corresponding abundances (Bioucas et al., 2012). LSMM is characterized by being a technique that estimates the proportions of the basic or pure components existing in each pixel of the image from the spectral response. The linear mixture approach has the advantage of being relatively easy to implement, and it also provides a physically meaningful measure that is portable across sensors and through time.

In regular or exploratory applications of the LSMM to remotesensing image data, three endmembers are initially assumed by many authors: green vegetation, bare soil, and shade/water (Shimabukuro et al., 2008; Zanotta et al., 2012). This assumption may not hold true for some natural scenes where a larger number of endmembers may be required for a proper modelling of the mixture problem.

The fraction images are the products generated by the algorithms of the LSMM. Usually the images are generated fraction of vegetation, soil and water/shade, which are generally presented in any image; fraction images can be seen as a way to reduce the dimensionality of data and also as a way to enhance information. (Ponzoni et al., 2010).

For the purpose of identifying unauthorized airstrips and trails, we apply the LSMM, to the bands 2, 3, and 4 of the images WMC-2/VRSS, with the software TerraAmazon.
The selection of the bands is carried out according to the similarity of the spectral bands between the VRSS-2 and CBERS-2, and bands 2, 3 and 4 are chosen for they have better discrimination for vegetation.

The identification of a pure pixel, which will extract the spectral signature of the elements of interest soil, vegetation and water/shade, is used to generate synthetic images representing respective fractions of each element. (See Figures 1, 2 and 3)

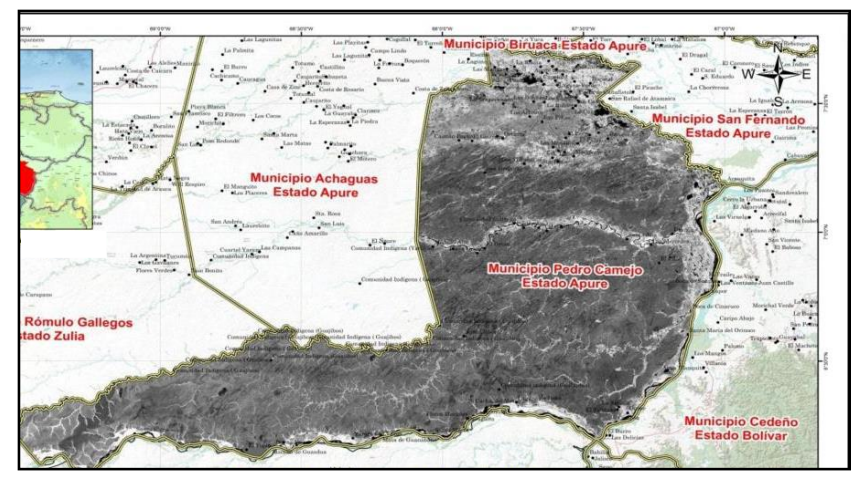

Figure 1. Image fraction of the soil component

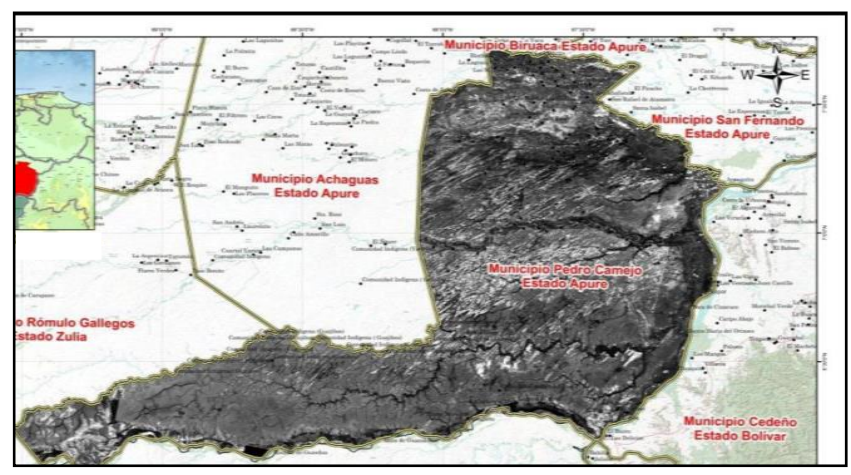

Figure 2. Image fraction of the vegetation component

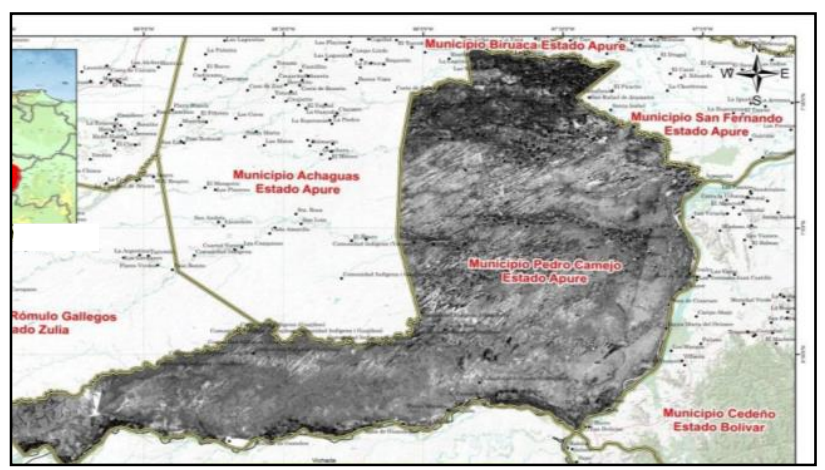

Figure 3. Image fraction of the water component

Once the thematic maps are obtained with the images fraction soil, vegetation and water; An analysis of each one was carried out; the map corresponding to the image fraction of the soil will consider more important, as it will allowed to discriminate more easily the elements of the geographical space as pattern, form and behavior. 


\subsection{GOBIA}

Geographic Object-Based Image Analysis is a promising alternative to identify probable illicit crops from images to remote sensors and auxiliary geographical data. Recently GOBIA has largely replaced the pixel-based analysis of remotely sensed imagery in high spatial resolution remote sensing field (Blaschke, 2010). GOBIA requires the images segmentation into homogeneous objects, which enables other attributes such as size, texture, shape and proximity relationships of objects to be used as descriptors of each object, in addition to allowing that other geographical data, such as the digital terrain model, are integrated into the image classification process; For this reason it is necessary to determine the data fusion and to combine in a semantic model, a set of classification rules that constitutes a form of representation of the knowledge.

VRRS-2 (1m) and OLI /LANDSAT-8 $(15 \mathrm{~m})$ of the study area is used to apply the GOBIA analysis. First the multiresolution segmentation approach in eCognition software is employed for the initial segmentation. Then, the data was parameterized according to the specific requirements: size, texture, shape, proximity, spectral and spatial values of the image, obtaining as a result, and segments adjusted to these attributes and incorporated into a multi-scale optimization routine.

Subsequent merging of small objects belonging to an individual crops complex seemed to be more straightforward than performing re-segmentations on objects that were considered to be too large. The segmentation performed using this scale parameter (250), with the same shape (0.1) and compactness (0.5) factors. For a comprehensive discussion of scale parameters, please refer Lang (2008) or Dragut et al.(2010). Subsequent steps, the smaller image objects were merged into larger objects on the basis of the chosen scale, color, and shape parameters, which define the growth in heterogeneity between adjacent image objects.

Following the various optimization steps, the resulting image segments were analyzed with respect to their spatial, spectral and textural parameters, then were classified into five classes: Potential areas for the planting of illicit crops, river with sediments, vegetation, shade of relief and clouds. (See Figure 9).

\subsection{MCE}

The general objective of the MCE is to help to choose the best alternative between a range of alternatives in an environment of competency and conflict criteria; and the objectives can be economic, environmental, social, institutional, technical and aesthetic. The key point of this process is the choice of alternatives, deciding on one or the other that presents a tendency towards a particular course of action. (Buzai, 2015).

Therefore, the application of the MCE was implemented as a method to identify susceptible areas to the propagation of illicit crops, for this, three variables are taken into consideration, and they are homogeneity, physiographic and socioeconomic variables.

\section{EXPERIMENTAL RESULTS}

\subsection{Unauthorized airstrips detection}

The detected airstrips were digitized with the attributes of geographic location (geographical coordinates) and track length. Subsequently for the purpose of determining whether or not they are authorized tracks in the study area, a superposition of the vectors of the aeronautical information of Venezuela were carried out, which in its content publishes the location Geographical (latitude and longitude) of the tracks registered and authorized in the country. See figure 4.

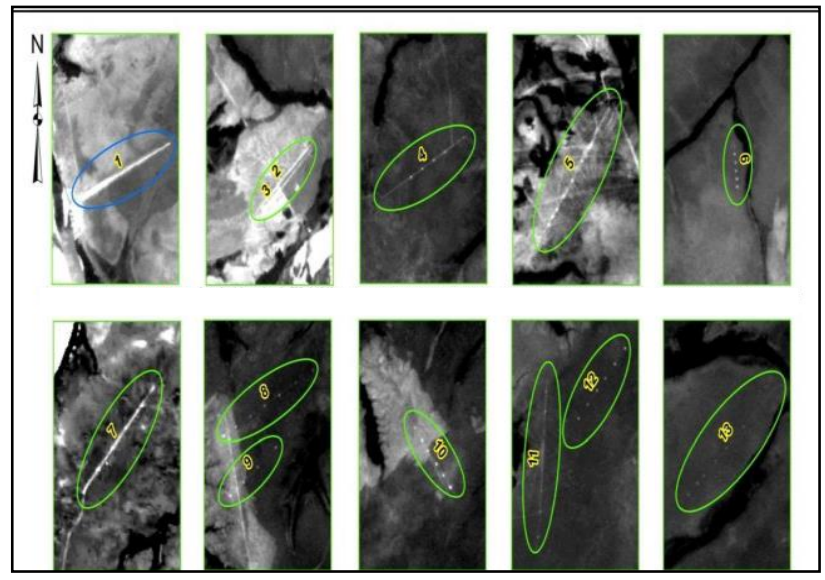

Figure 4. Unauthorized and authorized airstrips identified

36 airstrips are identified, of which 16 are authorized and 20 are unauthorized, and this information is verified with the state agencies.

In order to analyse the spatial pattern and the relation of the general characteristics of the illicit crops with the geographic space, a map was made (Figure 5) that allow to appreciate in more detail some airstrips identification

In the previous figure you can see some features or patterns that are generally taken into account for planting illicit crops, some of them are: unauthorized airstrips and roads to move the crops, gallery forests, water bodies and in this particular area the proximity to the Colombia border.

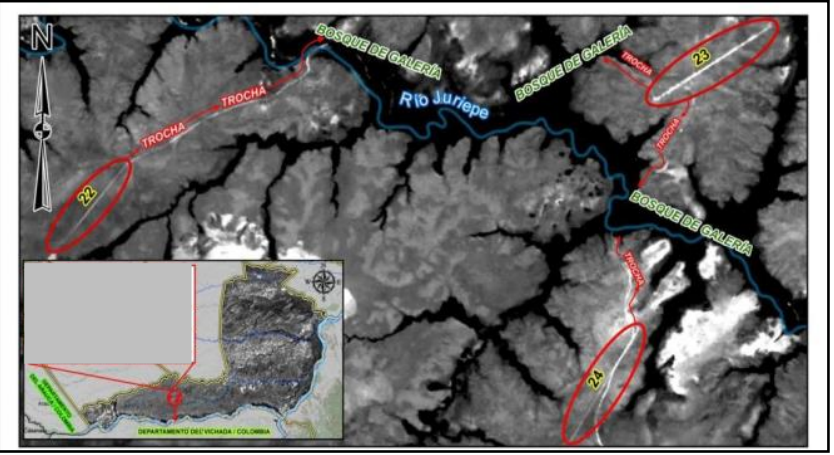

Figure 5. Unauthorized airstrips identified 


\subsection{Illicit crop detection}

After collecting all the important information given by the state agencies including: Armed forces, national anti-drug Office, Space agency, National Guard, etc, we proceed to apply the GOBIA technique and to obtain the areas prone to planting the illicit crops.

The fusion between LANDSAT-8 and VRSS-1 image is shown below in Figure 6.

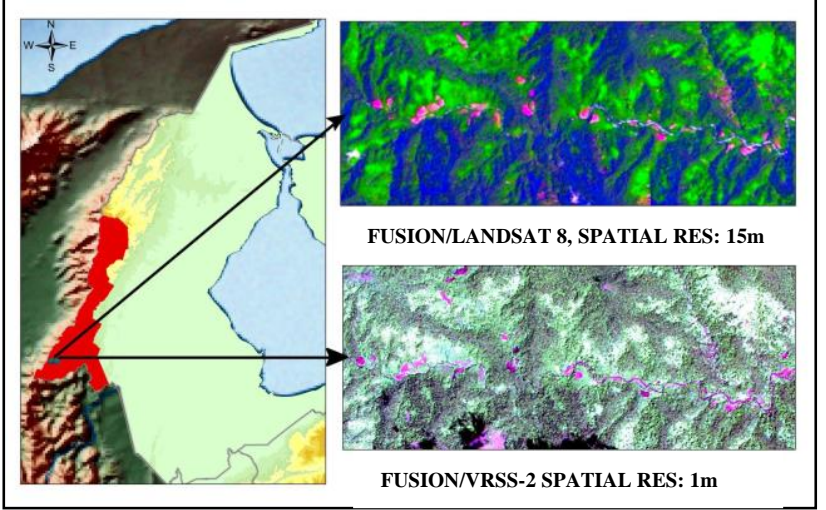

Figure 6. Selected area for the Gobia analysis.

Ecognition software is used to apply the technical GOBIA, the segmentation image is shown in the figure 7.

The segmentation process considered the parameters: scale, shape and compactness; the homogeneity criterion is also considered. Subsequently, several tests were conducted to determine the value that suits better for the identification of the interest elements, and 250 is set as scale value. The segmentation is performed using Multiresolution Segmentation algorithm, which considers many attributes including size, texture, shape, proximity, spectral and spatial values of the image

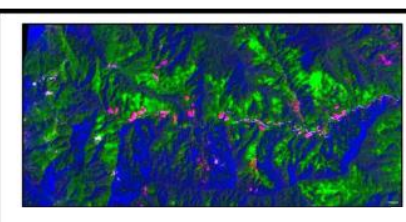

IMAGE WITHOUT SEGMENTATION
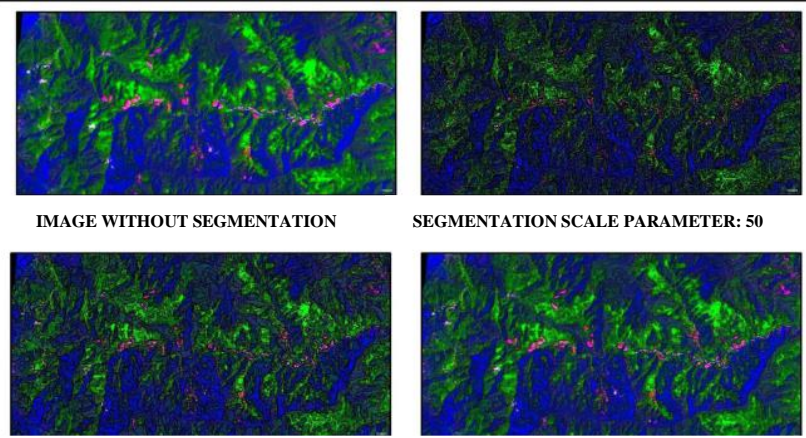

SEGMENTATION SCALE PARAMETER: 50

SEGMENTATION SCALE PARAMETER: 250

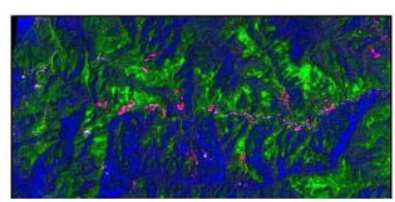

SEGMENTATION SCALE PARAMETER: 350

Figure 7. Segmentation test for Gobia analysis.

The thematic maps of interest classes is made, as shown in figure 8, which allow us to identify forty-two areas that correspond to the potential areas for planting illicit crops. In the same way the developed methodology allowed to identify the geographical location, as well as the number of affected hectares.

In the MCE part, three variables were taken into account, and they are: the slope of the terrain, the proximity to the border and the proximity to the watercourses (See Figure 9). 50\% weight is allocated to the slope variable of the terrain because generally this element is important for the people who sow these crops; the other $50 \%$ were distributed to the remaining variables.

Finally, this process is achieved by getting a set of subareas associated with a susceptibility index with respect to the establishment of illicit crops ranging from 0 to 100 percent, and then five categories are generated, which are assigned different ranks, thus allowing dividing the area of study according to different regions associated with their corresponding levels of susceptibility to the presence of illicit crops. See Figure 10.

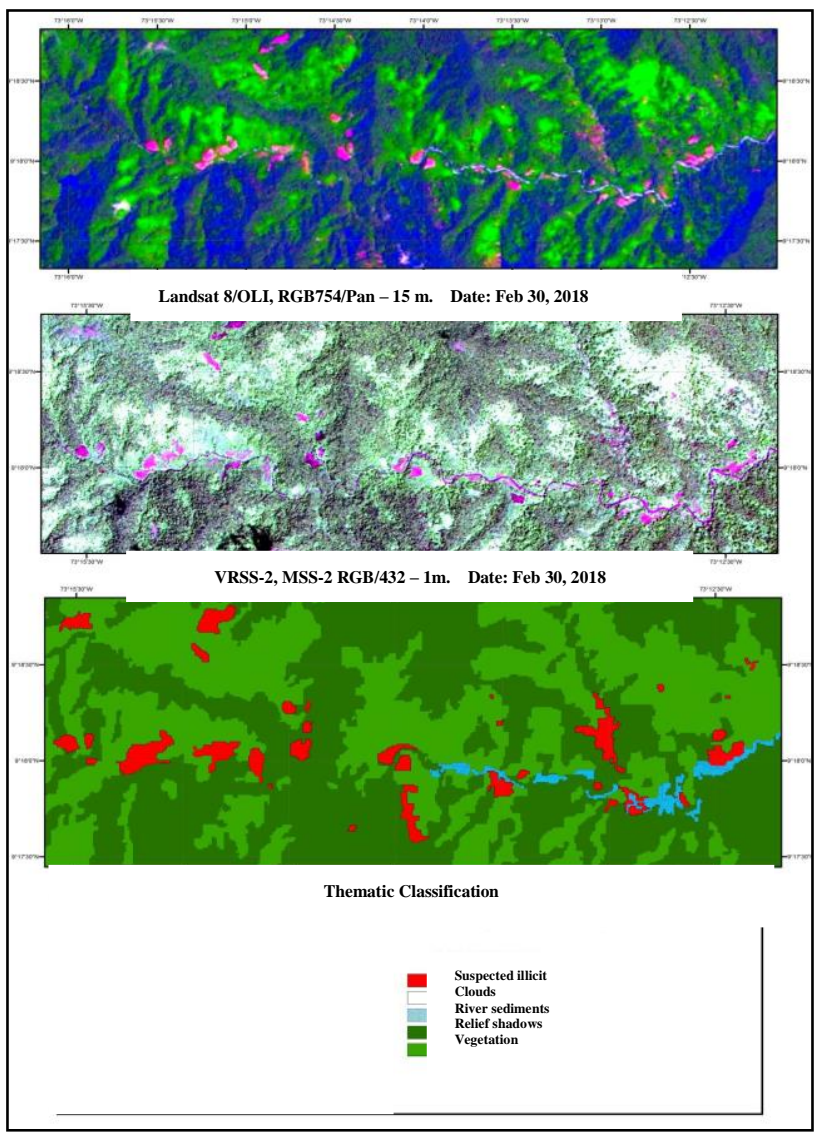

Figure 8. Potential areas for planting illicit crops
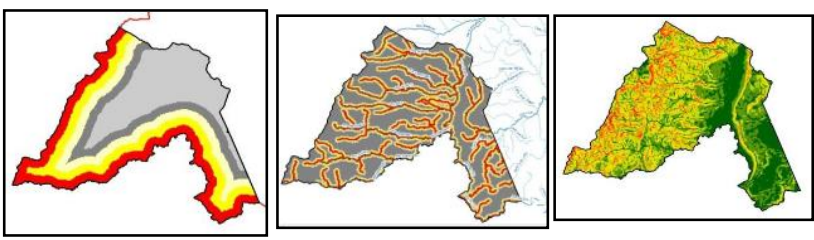

Figure 9. Left to right: slope of the terrain; Proximity to the Colombo-Venezuelan border and proximity to the water courses. 


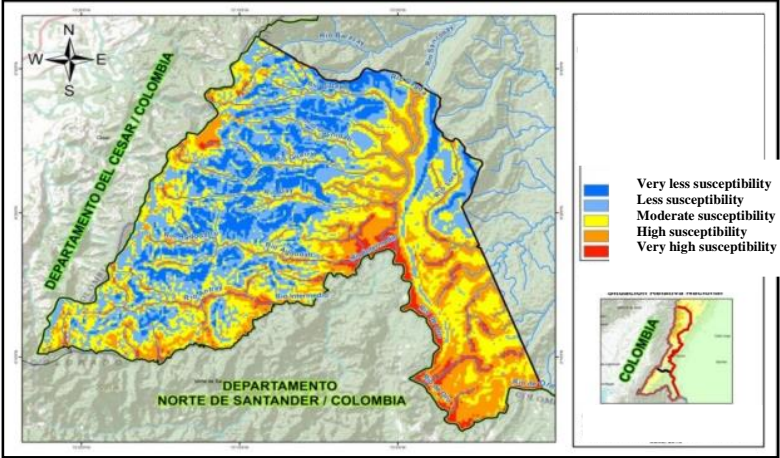

Figure 10. Map of susceptibility to the planting of illicit crops

\section{CONCLUSIONS}

In this research, different techniques of remote sensing data fusion were combined to detect illicit crops and unauthorized airstrips. The combination of LSMM, GOBIA and MCE fulfill the objectives of this research. The proposed method conducts data fusion in multiple level: signal, feature and decision, and it proves better performance compared to other similar approaches.

\section{ACKNOWLEDGMENTS}

This work is funded by State Grid Scientific Project 2016 (No. GCB17201600036) "Research on data processing theory and methods of the auxiliary lines selection based on satellite remote sensing image"

\section{REFERENCES}

Bioucas, J. et al., 2012. Hyperspectralunmixing overview: Geometrical, statistical, and sparse regression-based approaches, IEEE J. Sel. Topics Appl. Earth Observ. Remote Sens., 5 (2), pp. 354-379.

Blaschke, T., 2010. Object based image analysis for remote sensing. ISPRS Journal in Photogrammetry and Remote Sensing, 65(1), pp. 2-16.

Bogliacino, F. \& Naranjo, A., 2012. Coca leaves production and eradication: A general equilibrium analysis. Economics Bulletin. 32(1), pp. 382-397.

Buzai, G., 2015. Potentiality of the methodology of Multicriteria evaluation applied with geographic information systems. Geography, geotechnology and spatial analysis: Trends, methods and applications, Santiago de Chile, pp. 123128

Davalo,s E., 2016. New answers to an old problem: Social investment and coca crops in Colombia. International Journal of Drug Policy. 31, pp. 121-130.

Dragut, L., Tiede, D., and Levick, S., 2010. A tool to estimate scale parameter for multiresolution segmentation of remotely sensed data, Int. J. Geogr. Inf. Sci. 24, pp. 859-871.

Ehlers, M., Klonus, S., Åstrand, P., and Rosso, P. 2010. Multisensor image fusion for pansharpening in remote sensing. Int. Journal Image and Data Fusion, 1, (1), pp. 25-45.
Gamba P., 2013. Image and data fusion in remote sensing of urban areas: Status issues and research trends. Int. Journal Image Data Fusion, 5, (1), pp. 2-12.

Gustafsson, F., 2012. Statistical Sensor Fusion, 2nd ed. Studentlitteratur, Germany, pp. 48-65.

Huang, B. and Song, H., 2012. Spatiotemporal reflectance fusion via sparse representation. IEEE Trans. Geoscience of Remote Sensing, 50, (10), pp. 3707-3716.

Huang, B., Zhang, H., Song, H., Wang, J., and Song, C. 2013. Unified fusion of remote-sensing imagery: Generating simultaneously high-resolution synthetic spatial-temporalspectral earth observations. Remote Sensing. Letters, 4, (6), pp. 561-569.

Keshava, N. and Mustard, J., 2002. Spectral unmixing, IEEE Signal Process. Mag., 19, (1), pp. 44-57.

Khaleghi, B., Khamis, A., Karray, F., and Razavi, S., 2013. Multisensor data fusion: A review of the state of the art, Inform. Fusion, 14 (1), pp. 28-44.

Lang, S., 2008. Object-based image analysis for remote sensing applications: modeling reality - dealing with complexity, Berlin, Germany: Springer-Verlag, pp. 3-28.

Lin, C., Zhu, L., Wulimin., 2010. The research and realization of the forest information extraction based on the WorldView-2 image Science of Surveying and Mapping, 35(5), pp.205-207.

Liu, J., Wang, L., Yang, F. et al., 2015. Remote sensing estimation of crop planting area based on $\mathrm{HJ}$ time-series images. Transactions of the Chinese Society of Agricultural Engineering (Transactions of the CSAE), 31(3), pp.199-206.

Melgani, F. and Serpico S., 2002. A statistical approach to the fusion of spectral and spatio-temporal contextual information for the classification of remote-sensing images, Pattern Recognition Letters, 23, (9), pp. 1053-1061.

Moreno, S., Kraybill, R. \& Thompson, S., 2003. An econometric analysis of coca eradication policy in Colombia. World Development, 31(2), pp. 375-383.

Ponzoni, F. Shimabukuro, Y., 2010. Remote sensing in study of vegetation. São José dos Campos Brasil, Editora: Parêntese, pp. 157-179.

Reyes, L., 2011. Estimating the causal effect of forced eradication on coca cultivation in Colombian municipalities. Report MPRA (No. 33478), Colombia.

Schmitt, M and Xiang, Zhu., 2016. Data Fusion and Remote Sensing: An ever-growing relationship, IEEE Geoscience and remote sensing magazine, pp. 19-27.

Shimabukuro, Y., Almeida-filho, R., Kuplich T., and De Freitas R., 2008. Uses of Landsat TM fraction images to quantify the optical and SAR data relationships for land cover discrimination in the Brazilian Amazônia, International Journal in Geoinformatics, 4 (1), pp. 57. 
The International Archives of the Photogrammetry, Remote Sensing and Spatial Information Sciences, Volume XLII-3, 2018 ISPRS TC III Mid-term Symposium "Developments, Technologies and Applications in Remote Sensing", 7-10 May, Beijing, China

Venezuela Geographic Institute Simón Bolívar (IGVSB)., 2017.

Official Atlas of the Bolivarian Republic of Venezuela. Caracas.

Zanotta, D. and Haertel, V., 2012. Gradual land cover change detection based on multitemporal fraction images, Pattern Recognition, 45 (8), pp. 2927-2937.

Zhang, J., Chen, Y., Zhang, F., et al., 2012. Crops planting information extraction based on multi-temporal remote sensing images, Transactions of the Chinese Society of Agricultural Engineering (Transactions of the CSAE), 28 (2), pp.134-141.

Zhao, C., 2014. Advances of research and application in remote sensing for agriculture, Transactions of the Chinese Society of Agriculture Machinery, 45(12), pp. 277-293. 